\title{
Lévy processes and the Brazilian market
}

José Fajardo Barbachan*

Andrés Ricardo Schuschny**

André de Castro Silva***

\begin{abstract}
The present paper presents the Lévy processes used in the literature for the modeling of the returns of financial assets, which are generated by stable Paretian and hyperbolic distributions. Some properties of these distributions, especially the time-scale invariance, are analyzed. In the end, empirical evidence of the applicability of these processes is given for the modeling of Brazilian asset returns through Ibovespa, and the Telebrás and Petrobrás receipt. The data were collected between January 1st, 1995 and December 31st, 1998 (G1) and January 1st, 1996 and December 31st, 1997 (G2).
\end{abstract}

\section{Resumo}

No presente artigo apresentamos processos de Lévy usados na literatura para modelar os retornos dos ativos financeiros, estes processos são gerados pelas distribuiçōes Pareto-Estáveis e Hiperbólicas. Estudamos algumas propriedades destas distribuiçōes, em particular a propriedade da invariância da escala temporal. Por último apresentamos evidências empíricas da aplicabilidade destes processos para modelar retornos de ativos Brasileiros, para isto usamos o Ibovespa, o recibo da Telebrás e Petrobrás, na amostra usamos dados dos perodos de 1 은 de janeiro de 1995 a 31 de dezembro de 1998 (G1) e de $1^{\text {ㅇ }}$ de janeiro de 1996 a 31 de dezembro de 1997(G2).

Key Words: Hyperbolic Distribution, Stable Paretian Distribution, Fat Tails, Scale Invariance .

JEL Code: C52, G10 .

\footnotetext{
" Universidade Católica de Brasília, SGANi 916, Asa Norte, DF-BRAZIL

** Universidad Nacional de Quilmes, Roque Saenz Pena 180, Bernal, Pcia. de Buenos Aires, Argentina

${ }^{* * *}$ Chicago University
}

Brazilian Review of Econometrics Rio de Janeiro v.21, $\mathrm{n}^{\mathrm{O}}$ 2, pp.263-289 Nov.2001 
Lévy processes and the Brazilian market

\section{Introduction.}

The hypothesis of normality of assets returns has been criticized by many authors. Empirically, this hypothesis does not apply to most financial assets since the distribution of returns has heavier tails than those of normal distribution as well as greater concentration around the mean. Among the studies in which this hypothesis is criticized, we have "The behavior of stock market prices" by Fama (1965), and "On the distributions of stock price differences" by Mandelbrot and Taylor (1967). These authors suggested stable Paretian distributions ${ }^{1}$ as an alternative to modeling the returns of financial assets. Some properties of these distributions (the timescale invariance, for instance) are remarkably interesting and allow us to analyze the behavior of financial markets. The downside of these distributions is that they have very heavy tails.

On the other hand, we have the hyperbolic distributions introduced by Barndorff-Nielsen (1977) to model the shape of sand particles formed by the wind and water. This type of distribution is currently used in finance due to its good adherence to returns data of several assets (see Eberlein and Keller (1995) and Kuchler et al. (1994)).

Apart from the models mentioned above, there are other models that assess the stylized facts of financial series. Among discreet models, it is worthwhile mentioning ARCH and GARCH models. For applications of these models with Brazilian data, see Issler (1999), Duarte and Mendes (1999), Mazucheli and Migon (1999) and Pereira

\footnotetext{
${ }^{1}$ The term derives from economist Vilfredo Pareto, who first used this type of distribution for modeling the income distribution of an agent. The term "Stable" relates to probability. For further details, see Mandelbrot (1997).
} 
José Fajardo Barbachan, Andrés Ricardo Schuschny and André de Castro Silva

et al. (1999). Among continuous models, we have Lévy models, including the models of stochastic volatility and jumps, which are empirically less used due to their mathematical complexity. The models outlined in the present study fit the Lévy model. There are no results based on this model using Brazilian data. Therefore, we believe our paper offers some evidence of the possible application of this model to the assessment of the Brazilian Market.

The article is organized as follows: In section 2, we present the stable Paretian distributions; in section 3, we deal with the property of scale invariance and empirical evidence of its use with data obtained in Brazil. Section 4 is concerned with hyperbolic distributions, the estimation of parameters related to the adherence of Brazilian data to this model and the analysis of scale invariance. Section 5 shows our conclusions, while Section 6 consists of an Appendix.

\section{Stable distributions and Lévy flights.}

\subsection{Introduction.}

Suppose a stochastic process characterized by a one-dimensional random walk whose jumps are independent and identically distributed with a probability $p(x)$. A possible question would be: When probability $P_{n}(x)$ of the walk gets to position $x$ after $n$ steps $\left(x=x_{1}+. .+x_{n}\right)$, is the same as that of $p(x)$ in less than a scale factor? In other words, could there be a $p(x)$ that produces a stochastic process with a self-similar walk trajectory? The answer is "yes". If $p(x)$ turns out to be a normal distribution with mean $\mu$ and variance $\sigma, P_{n}(x)$ will also be a normal distribution with mean $\mu_{n}=n \mu$ and variance $\sigma_{n}=n \sigma$. However, at the beginning of the century, Lévy (1937) showed that this was only a particular case and that other solutions which allow self-similar walk trajectories exist. 
Lévy processes and the Brazilian market

\subsection{Stable distributions.}

The distribution of a sum of independent random variables is the convolution of their distributions. This means:

$$
P_{n}(x)=\int_{-\infty}^{+\infty} P_{n-m}(x-y) P_{m}(y) d y \quad 0 \leq m \leq n
$$

In practice, as the Fourier transform of a convolution of distributions is the product of the transforms of each distribution, we had better work with characteristic functions. The characteristic functions are defined as the Fourier transform of these distributions. As a result, we have:

$$
\hat{P}_{n}(k)=\hat{P}_{n-m}(k) \cdot \hat{P}_{m}(k) \quad \text { with } \hat{P}(k)=<e^{i k x}>=\int_{-\infty}^{+\infty} e^{i k x} P(x) d x
$$

Where $k$ the associated conjugate variable. Lévy showed that the general solution to this equation has the following form ${ }^{2}$ :

$$
\hat{P}_{n}(k)=e^{-n|k|^{\beta}}
$$

${ }^{2}$ The most general expression of a Lévy-stable distribution is:

$$
\hat{P}_{n}(k)=\exp \left(-\mu k-n|k|^{\beta}(1+i \alpha \operatorname{sgn}(k) \omega(|k|, \beta))\right)
$$

where $i=\sqrt{-1}, \omega(|k|, \beta)=\tan \left(\frac{\pi \beta}{2}\right)$ if $\beta \neq 1$, and $\omega(|k|, \beta)=\frac{\pi}{2} \ln (|k|)$, if $\beta=1$, with $\beta \in(0,2]$, and $\alpha \in[-1,+1] . \beta$ is the critical exponent, which determines the tail shape. $\alpha$ measures the level of symmetry of the distribution. If negative, the distribution slants to the right; if positive, it slants to the left; and if it is zero, the distribution is symmetric in relation to mean $\mu$. 
José Fajardo Barbachan, Andrés Ricardo Schuschny and André de Castro Silva

The distributions with this type of characteristic function are called Lévy-stable distributions and correspond to the stochastic processes known as Lévy flights. In Finance these distributions are also called stable Paretian distributions. In what follows we will use these two terms as synonymous.

\subsection{Lévy Flight Properties.}

(i) By definition, the distribution function is:

$$
P_{n}(x)=\frac{1}{2 \pi} \int_{-\infty}^{+\infty} e^{-n|k|^{\beta}} e^{-i k x} d k
$$

(ii) It is possible to show that $|\hat{p}(k)| \leq \hat{p}(k=0)=1$, in fact:

$$
\begin{aligned}
|\hat{p}(k)| & =\left|\int_{-\infty}^{+\infty} p(x) e^{i k x} d x\right| \leq \int_{-\infty}^{+\infty}\left|e^{i k x}\right| p(x) d x \\
& =\int_{\infty}^{+\infty} p(x) d x=1 \equiv \hat{p}(k=0)
\end{aligned}
$$

Observe that studying $p(k)$ for values close to the origin is similar to studying the distribution function for values of $x \longrightarrow \infty$.

(iii) For every characteristic function, if we develop exponential $e^{-i k x}$, the moment of order $q$ of the distribution is:

$$
<x^{q}>=(-1)^{q} \frac{\partial \hat{p}(k=0)}{\partial k^{q}} \quad q=0,1,2, \cdots
$$

So, by using 3 and the Chain Rule, we obtain: $\hat{P}_{n}(k)=\hat{p}^{n}(k)$ and as $\hat{p}(k=0)=1$ therefore $\left.\left\langle x^{q}(n)\right\rangle=n<x^{q}\right\rangle$. 
(iv) By using $\hat{p}(k) \equiv e^{-|k|^{\beta}}$, it is evident that $\left\langle x^{2}>\right.$ only exists if $\beta=2$. For all $\beta \in[0.2)$, the second-order moment of these distributions is infinite. From the sample, it is not possible to show whether the variance of a distribution is finite because as the sample size is finite, we will always have a defined estimator for the variance of the distribution. By using finite time series, the moments associated with the distribution are affected by the values that float around the center of the distributions and not by the tails.

(v) If the distribution is stable, we have potential tails, in fact, when $|k| \approx 0$ :

$$
\begin{aligned}
P_{n}(x) & =\frac{1}{2 \pi} \int_{-\infty}^{+\infty} e^{-n|k|^{\beta}} e^{-i k x} d k=\frac{1}{\pi} \int_{0}^{+\infty} e^{-n|k|^{\beta}} \cos (k x) d k= \\
& =\frac{1}{\pi|x|} \int_{0}^{+\infty} \exp \left(\frac{-n|\nu|^{\beta}}{|x|^{\beta}}\right) \cos (\nu) d \nu \\
& \approx \frac{1}{\pi|x|} \int_{0}^{+\infty}\left(1-\frac{n|\nu|^{\beta}}{|x|^{\beta}}\right) \cos (\nu) d \nu \approx \\
& \approx-\frac{n}{\pi|x|^{\beta+1}} \int_{0}^{+\infty}|\nu|^{\beta} \cos (\nu) d \nu \\
& =-\frac{n}{\pi|x|^{\beta+1}} \lim _{\epsilon \rightarrow 0} \int_{0}^{+\infty} e^{-\epsilon \nu}|\nu|^{\beta} \cos (\nu) d \nu= \\
& =\Gamma(1+\beta) \sin \left(\frac{\pi \beta}{2}\right) \frac{n}{\pi|x|^{\beta+1}}
\end{aligned}
$$

with $\Gamma(z)=\int_{0}^{+\infty} u^{z-1} e^{-u} d u$, for $z>0$.

Therefore:

$$
P_{n}(x) \approx|x|^{-\psi} \quad \text { if } \quad x \rightarrow \infty(\text { or } k \rightarrow 0)
$$


José Fajardo Barbachan, Andrés Ricardo Schuschny and André de Castro Silva

(vi) For instance, we can show that Cauchy distribution corresponds to the case $\beta=1$, and therefore we have:

$\hat{P}_{n}(k)=e^{-n|k|}$ and $\hat{p}(k)=e^{-|k|}$, then we obtain:

$P_{n}(x)=\frac{1}{\pi n} \cdot \frac{1}{1+\left(\frac{x}{n}\right)^{2}}=\frac{1}{n} p\left(\frac{x}{n}\right)$

The parameter that characterizes Lévy-stable distributions is $\beta$ and describes the roughness of the time series. When $\beta=2$, (Gaussian case) the correlation between increments is null.

$$
\hat{P}_{n}(k) \equiv \hat{p}(k)^{n}=e^{-n|k|^{\beta}}
$$

\section{Lévy Flights and Financial Index.}

In this section, we analyze the evolution of Ibovespa, drawing attention to the fact that this index can be modeled by a symmetric Lévy flight. The same applies to other financial indices. As descriptive variable, we will use the return ${ }^{3}$ calculated for different time intervals $\Delta t$, i.e.

$$
r(t)=\frac{I(t)-I(t-\Delta t)}{I(t-\Delta t)} \quad \Delta t \sim 1,5,10,20,30 \text { days }
$$

Where $I(t)$ is the value of Ibovespa at time $t$. To perform the quantitative analysis of data, first we are going to determine the probability $P(r)$ of returns for different time intervals. Figure 1 shows the graph for $P(r)$ using different values of $\Delta t$. We can observe that the tails of these distributions become 'loose' as $\Delta t$ interval increases. If we choose a greater interval to calculate the relative returns, the chances to have larger returns (positive or negative) also increase.

\footnotetext{
${ }^{3}$ Return is synonymous with rate of return.
} 
Lévy processes and the Brazilian market

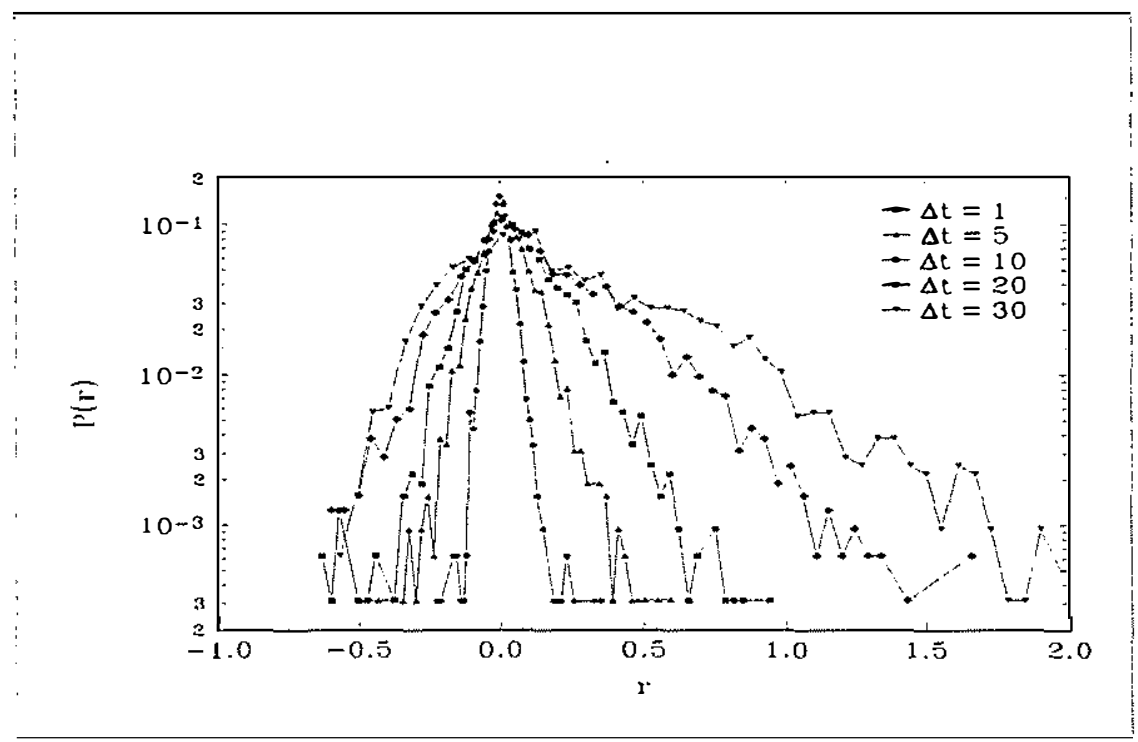

Figure 1: Distribution of Returns

To calculate the characteristic exponent of stable distribution $(\beta)$, we estimated the probability of returning to origin $P(r=0)$ as function of $\Delta t$ (this approach was introduced by Mantegna (1995)). This value can be affected by the finiteness of data. Figure 2 shows $P(r=0)$ as function of $\Delta t$, on a $\log -\log$ scale, using different values for $\Delta t$. We can adjust a straight line with a $-0,63$ inclination to these data. Up to the first $50 \Delta t$ values, the correlation coefficient of the adjustment is 0,985 . As expected, we have a nonGaussian behavior. A test could be performed to check whether or not the difference is significant; however, since this is well-documented in literature, the test will not be carried out. The reason for the use of the probability of return to the origin as the basis for the calculation of characteristic exponent $\beta$ is that if we theoretically calculate this probability as function of $\Delta t$, we obtain the power law, shown in Figure 2. In fact, if we take the definition of distribution: 
José Fajardo Barbachan, Andrés Ricardo Schuschny and André de Castro Silva

$$
\begin{aligned}
P_{n}(r, \Delta t) & =\frac{1}{2 \pi} \int_{-\infty}^{+\infty} e^{-n \Delta t|k|^{\beta}} e^{-i k r} d k \\
& =\frac{1}{\pi} \int_{0}^{+\infty} e^{-n \Delta t|k|^{\beta}} \cos (k r) d k
\end{aligned}
$$

By changing variables $\left(u=n \Delta t|k|^{\beta}\right)$ and using the gamma function defined by $\Gamma(z)=\int_{0}^{+\infty} u^{z-1} e^{-u} d u$, for $z>0$, we conclude that:

$$
P_{n}(0, \Delta t)=\frac{1}{\pi} \int_{0}^{+\infty} e^{-n \Delta t|k|^{\beta}} d k=\frac{\Gamma\left(\frac{1}{\beta}\right)}{\pi \beta(n \Delta t)^{\frac{1}{\beta}}}
$$

This power law ${ }^{4}$ allows us to calculate the critical exponent of the stable distribution that better suits the process that generates the market data. Another way to calculate this exponent is to use property (5); however, the data set is not enough to analyze the behavior of the distribution far from the origin.

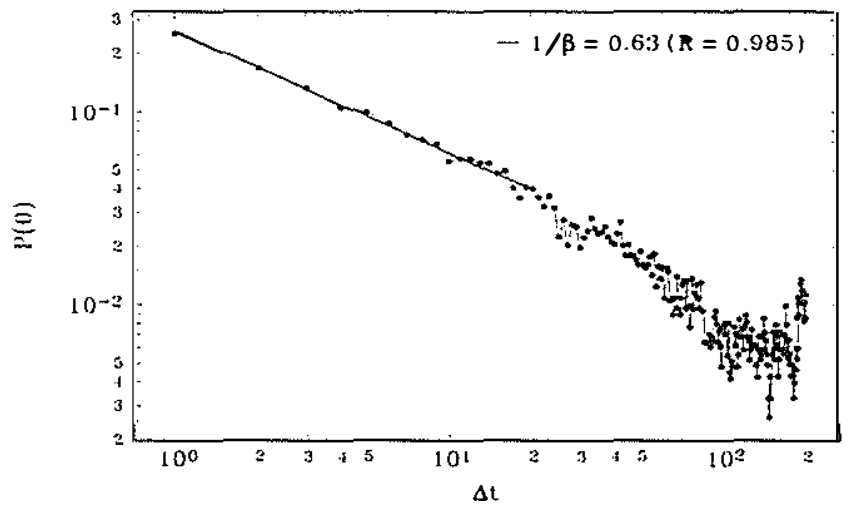

Figure 2: Log/Log Graph

\footnotetext{
${ }^{4}$ See appendix.
} 


\subsection{Scale Invariance.}

Some systems have something we call scale-free behavior; this means some kind of symmetry is preserved among the scales where systems are developed. This scale invariance occurs when some observation of the system presents a power law.

As the financial market is subject to accurate iteration rules, we verify there is room for scale invariance. Therefore, we will analyze some statistical properties of the stock market. We will see that the evolution of the financial assets returns or index is governed by a Lévy-stable distribution.

After the characteristic exponent is known, we can observe that the different distributions are similar except for a scale factor, which means that, as previously mentioned, the stochastic process does not vary with time-scale transformations. To verify this property, it is possible to rescale all distributions to $\Delta t$, submitting the variables to the following transformation:

$$
r_{s}=\frac{r}{(\Delta t)^{1 / \beta}} \quad \text { e } \quad P_{n, s}\left(r_{s}, \Delta t\right)=\frac{P_{n}(r, \Delta t)}{(\Delta t)^{-1 / \beta}}
$$

Figure 3 shows that, except for large width events, the scaled distributions are superposed on a single distribution. This validates the thesis that financial markets, in general, have a self-similar timescale. This property gives us evidence that, in these markets, it is not possible to define a privileged time-scale in the system. A possible explanation could be the heterogeneity through which expectations and decisions are formed and the impact they have on price evolution. A set of negotiators that operate with different restrictions and time horizons produce a scale-free market dy namics. Perhaps the microstructure that sustains the existence of the market gives rise to a kind of amalgamation of relaxed times that follow the impact caused by new information. 
José Fajardo Barbachan, Andrés Ricardo Schuschny and André de Castro Silva

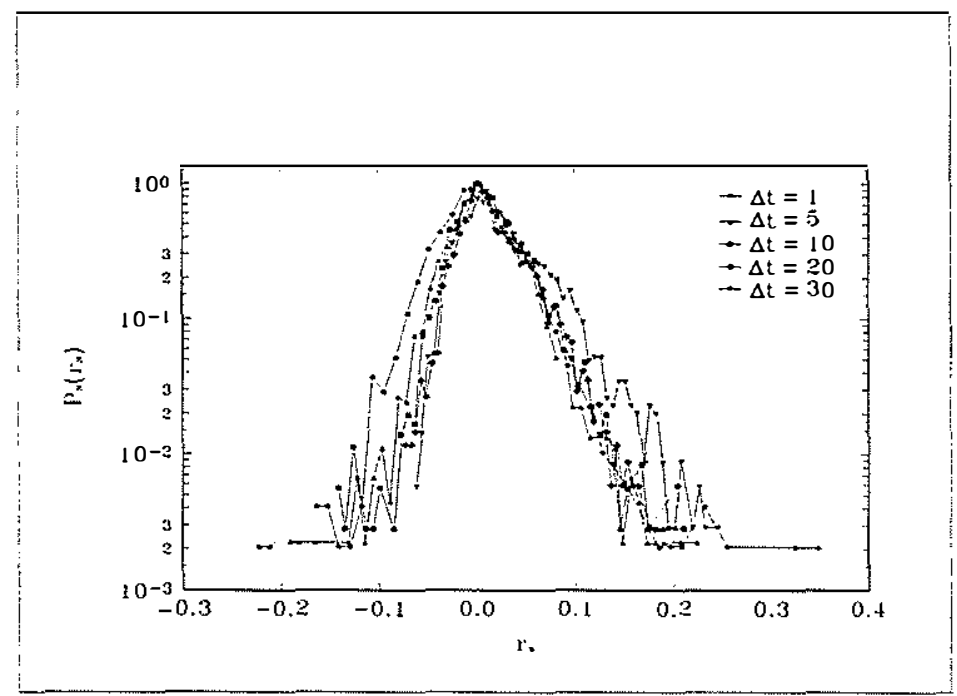

Figure 3: Graph of returns with the new scale

In figure 2 , we have problems with $\Delta t=30$, which means that it is hard to produce the desired scale. However, as this $\Delta t$ is very large, we have no potential behavior.

The downside of these distributions is the presence of 'fat' tails. Some studies using hyperbolic distributions have been presented in literature to improve data adjustment. Next, we will analyze the data of some assets and index by using these distributions.

\section{Hyperbolic Analysis.}

\subsection{Hyperbolic distribution.}

This section presents the hyperbolic model, showing the parameter estimates. Also, it shows that the hyperbolic model offers a good fit of data and a better fit than the normal distribution. 
The hyperbolic distribution function is built in such a way to present a linear histogram when constructed on a log-log scale. The curve with such behavior is the hyperbola, which is expressed as

$$
y=-\alpha \sqrt{1+x^{2}}+\beta x,
$$

with asy mptotes of $x \rightarrow+\infty$ and $x \rightarrow-\infty$ respectively given by $y=\phi x$ and $y=-\gamma x$ where $\phi=\alpha+\beta$ and $\gamma=\alpha-\beta$. The domain of $\phi$ and $\gamma$ is $(0, \infty)$. The probability function correspondent to (13) is given by

$$
a(\alpha, \beta) \exp \left(-\alpha \sqrt{1+x^{2}}+\beta x\right),
$$

where $a(\alpha, \beta)$ is a constant added to produce

$$
\int_{-\infty}^{+\infty} a(\alpha, \beta) \exp \left(-\alpha \sqrt{1+x^{2}}+\beta x\right) d x=1 .
$$

Now, take $\alpha=\frac{\phi+\gamma}{2}$ and $\beta=\frac{\phi-\gamma}{2}$, with this parametrization we can rewrite (14),

$$
a(\phi, \gamma) \exp \left(-\frac{1}{2}\left\{\phi\left[\sqrt{\left(1+x^{2}\right)}-x\right]+\gamma\left[\sqrt{\left(1+x^{2}\right)}+x\right]\right\}\right),
$$

or, by multiplying the exponent in (15) by $\left(\sqrt{1+x^{2}}+\right.$ $x) /\left(\sqrt{1+x^{2}}+x\right)$

$$
a(\phi, \gamma) \exp \left(-\frac{1}{2}\left\{\phi\left[\sqrt{\left(1+x^{2}\right)}+x\right]^{-1}+\gamma\left[\sqrt{\left(1+x^{2}\right)}+x\right]\right\}\right)
$$


José Fajardo Barbachan, Andrés Ricardo Schuschny and André de Castro Silva

To determine constant $a(\phi, \gamma)$, we change the following variable: $\nu(x)=\sqrt{\left(1+x^{2}\right)}+x$, as $\nu(-\infty)=0$ and $\nu(+\infty)=+\infty$, we have

$$
\begin{gathered}
\frac{1}{2} \int_{0}^{+\infty}\left(1+\nu^{-2}\right) \exp \left(-\frac{1}{2}\left(\phi \nu^{-1}+\gamma \nu\right)\right) d \nu= \\
=\int_{-\infty}^{+\infty} \frac{1}{2}\left(1+\frac{1}{\left(\sqrt{\left(1+x^{2}\right)}+x\right)^{2}}\right)\left(1+\frac{x}{\sqrt{\left(1+x^{2}\right)}}\right) \\
\exp \left(-\frac{1}{2}\left(\phi \nu(x)^{-1}+\gamma \nu(x)\right) d x\right. \\
=\int_{-\infty}^{+\infty} \exp \left(-\frac{1}{2}\left(\phi \nu(x)^{-1}+\gamma \nu(x)\right) d x .\right.
\end{gathered}
$$

However the expression in (17) is equal to $\kappa K_{1}(\kappa) / \omega$, where

$$
\kappa=\sqrt{\phi \omega} \text { and } \omega=\frac{1}{\phi^{-1}+\gamma^{-1}}
$$

and $K_{1}$ is the modified Bessel function of the third kind and index 1 (see appendix). Thus, $a(\phi, \gamma)=\frac{\omega}{\kappa K_{1}(\kappa)}$, where $\kappa$ and $\omega$ are given by (18). Therefore, once $\phi$ and $\gamma$ are given, the probability distribution function is

$$
f(x ; \phi, \gamma)=\frac{\omega}{\kappa K_{1}(\kappa)} \exp \left(-\frac{1}{2}(\phi+\gamma)\left(\sqrt{1+x^{2}}\right)+\frac{1}{2}(\phi-\gamma)(x)\right)
$$


By including a location parameter $\mu$ and a positive scale factor $\delta$, we obtain, from (19),

$$
\begin{aligned}
& f(x ; \phi, \gamma, \mu, \delta)=\frac{\omega}{\delta \kappa K_{1}(\delta \kappa)} \\
& \quad \exp \left(-\frac{1}{2}(\phi+\gamma)\left(\sqrt{\delta^{2}+(x-\mu)^{2}}\right)+\frac{1}{2}(\phi-\gamma)(x-\mu)\right) .
\end{aligned}
$$

Where we write $\phi$ and $\gamma$ in the place of $\phi / \delta$ and $\gamma / \delta$,respectively. In equation (20), function $\ln f(. ; \phi, \gamma, \mu, \delta)$ describes a hyperbola with slant asy mptotes $\phi$ and $\gamma$. If we turn back to the initial parametrization, the hyperbolic probability distribution function will be given by

$$
\begin{array}{r}
h y p(x ; \alpha, \beta, \delta, \mu)=\frac{\sqrt{\alpha^{2}-\beta^{2}}}{2 \alpha \delta K_{1}\left(\delta \sqrt{\alpha^{2}-\beta^{2}}\right)} \\
\exp \left(-\alpha \sqrt{\delta^{2}+(x-\mu)^{2}}+\beta(x-\mu)\right) .
\end{array}
$$

The hyperbolic distribution was proposed by Barndorff-Nielsen (1977). It was initially presented to model the movement of sand particles cast by winds with a continuous speed. That article suggests other applications for the hyperbolic distribution, such as financial data. Barndorff-Nielsen (1978) presents an application for the structure of income distribution. Eberlein and Keller (1995) were the first ones to use this distribution in finance.

The hyperbolic distribution is able to describe a considerable number of empirical distributions with asy mmetric behavior or with 'fat' tails. Observe that this distribution has semi-heavy tails, that is

$$
h y p(x ; \alpha, \beta, \delta, \mu) \sim e^{-( \pm \alpha-\beta) x} \text { when } x \rightarrow \pm \infty
$$


José Fajardo Barbachan, Andrés Ricardo Schuschny and André de Castro Silva

However, contrary to other distributions, the parameters of the hyperbolic distribution are apparently not able to provide much information on the distribution format. One way to study the form of distribution, suggested by Barndorff-Nielsen et al. (1985), is to write $h y p(x ; \chi, \xi, \delta, \mu)$ using the following parameters

$$
\xi=\left(1+\delta \sqrt{\alpha^{2}-\beta^{2}}\right)^{-\frac{1}{2}}, \quad \text { and } \quad \chi=\xi \beta / \alpha
$$

Such parametrization has the advantage of making $\xi$ and $\chi$ invariant even with scale and location transformations. The domain for parameter variation is given by

$$
0 \leq|\chi|<\xi<1
$$

This domain is known as shape triangle and is represented in figure (4). The symbols $N, N_{1}^{-}, L$, and $E$ on the borders respectively indicate normal distribution, generalized inverse Gaussian distribution, with index $\lambda=1$, the Laplace distribution and the exponential.

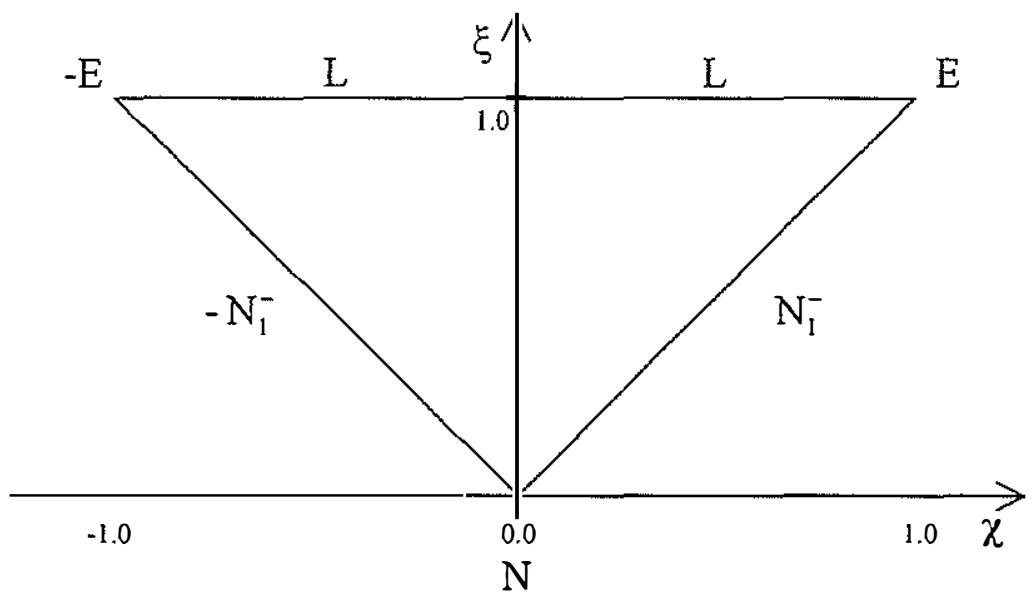

Figure 4: Variation domain of paramaters 
All these distributions are limits to the hyperbolic distribution. This allows us to understand $\xi$ and $\chi$ asymptotically as kurtosis and asymmetry.

To obtain the distribution limits, Barndorff-Nielsen et al. (1985) chose $\delta$ so that the variance of the hyperbolic distribution could be equal to 1 and the mean could equal 0 . With these simplifications, the distribution assumes the following shape

$$
h y p(x ; \xi, \chi)=a(\xi, \chi) \exp \left(-b(\xi, \chi)\left[\sqrt{1-(\chi / \xi)^{2}+z^{2}}-(\chi / \xi)^{2} z\right]\right)
$$

where

$$
\begin{gathered}
z=\frac{x}{\xi^{-1}-1}+\frac{\chi}{\xi}, \\
a(\xi, \chi)=\left[2\left(\xi^{-1}-1\right) K_{1}\left(\xi^{-2}-1\right)\right]^{-1}, \text { and } \\
b(\xi, \chi)=\frac{\xi^{-2}-1}{1-\chi / \xi} .
\end{gathered}
$$

With $\xi \rightarrow 0$, we have the normal distribution

$$
\frac{1}{\sqrt{2 \pi}} \exp \left(-\frac{1}{2 x^{2}}\right) \text {. }
$$

With $\xi \rightarrow 1$, we have the Laplace distribution

$$
\exp \left(-\frac{2}{1-\chi^{2}}(|x|-\chi x)\right)
$$

For $\chi=0$, in particular, we have $e^{-2|x|}$. For $|\chi| \rightarrow 1$, we obtain the exponential distribution. The generalized inverse Gaussian distribution is obtained by $\chi \rightarrow \pm \xi$. The moment-generating function of the hyperbolic distribution is given by

$$
M(t)=e^{\mu t} \frac{\sqrt{\alpha^{2}-\beta^{2}}}{K_{1}\left(\delta \sqrt{\alpha^{2}-\beta^{2}}\right)} \frac{K_{1} \delta \sqrt{\alpha^{2}-(\beta+t)^{2}}}{\sqrt{\alpha^{2}-(\beta+t)^{2}}} \quad|\beta+t|<\alpha .
$$


José Fajardo Barbachan, Andrés Ricardo Schuschny and André de Castro Silva

Eberlein et al.(1998) obtain this expression by using (21) with $\mu=0$. Therefore, we have $a(\alpha, \beta, \delta)=\frac{\sqrt{\alpha^{2}-\beta^{2}}}{2 \alpha \delta K_{1}\left(\delta \sqrt{\alpha^{2}-\beta^{2}}\right)}$.

$\operatorname{For}|\beta+t|<\alpha$

$$
\begin{aligned}
M(t) & =\int e^{t x} f(x ; \alpha, \beta, \delta, 0) d x \\
& =a(\alpha, \beta, \delta) \int \exp \left(-\alpha \sqrt{\delta^{2}+x^{2}}+(\beta+t) x\right) d x \\
& =\frac{a(\alpha, \beta, \delta)}{a(\alpha, \beta+t, \delta)} \\
& =\frac{\sqrt{\alpha^{2}-\beta^{2}}}{K_{1}\left(\delta \sqrt{\alpha^{2}-\beta^{2}}\right)} \frac{K_{1} \delta \sqrt{\alpha^{2}-(\beta+t)^{2}}}{\sqrt{\alpha^{2}-(\beta+t)^{2}}} .
\end{aligned}
$$

Thus, all the moments of the function exist. For the expected value and for variance, we obtain,

$$
\begin{gathered}
E[X]=\mu+\frac{\delta \beta}{\sqrt{\alpha^{2}-\beta^{2}}} \frac{K_{2}(\zeta)}{K_{1}(\zeta)}, \text { and } \\
\operatorname{Var}[X]=\delta^{2}\left(\frac{K_{2}(\zeta)}{\zeta K_{1}(\zeta)}+\frac{\beta^{2}}{\alpha^{2}-\beta^{2}}\left[\frac{K_{3}(\zeta)}{K_{1}(\zeta)}-\left(\frac{K_{2}(\zeta)}{K_{1}(\zeta)}\right)^{2}\right]\right),
\end{gathered}
$$

where $X \sim h y p(x ; \alpha, \beta, \delta, \mu)$ and $\zeta=\delta \alpha^{2}-\beta^{2}$. In the following section, we will see how we can use this distribution to model the returns. 


\subsection{Return model.}

In this context, let us suppose that the price of assets are modeled by the following equation

$$
S_{t}=e^{X_{t}}
$$

Where $\left\{X_{t}\right\}_{t \geq 0}$ is a Lévy process generated by the hyperbolic distribution. Now we can define returns $R_{t}$ as

$$
R_{t}=\log \frac{S_{t}}{S_{t-1}} \approx \frac{S_{t}}{S_{t-1}}-1,
$$

We observe that the right-hand side approximation occurs when the difference between today's and yesterday's closing price is small. Observe that if we actually define

$$
R_{t}^{*}=\frac{S_{t}}{S_{t-1}}-1
$$

we would have

$$
R_{t}^{*}-R_{t}=\frac{1}{2 R_{t}^{2}}+\frac{1}{6 R_{t}^{3}}+\cdots
$$

As most authors do, we will use the one that corresponds to a continuous composition. The main reason is that the return in $n$ periods would be given by:

$$
R_{t+1}+\cdots+R_{t+n}=\log \left(\frac{S_{t+n}}{S_{t}}\right),
$$

this is not satisfied by $R_{t}^{*}$. In addition, we should not forget that for continuous time processes, the continuously compound rate of 
return is a natural choice. If the prices were lognormal, that is, if $X_{t}$ were a normal variable or more specifically a standard Brownian motion, we would have

$$
R_{t}=X_{t}-X_{t-1} \sim N(0.1)
$$

Therefore, lognormality of prices would imply normality of returns, so we need another model for prices or for returns. Our candidate to be tested will be the hyperbolic Lévy motion, that is, the Lévy process generated by the hyperbolic distribution. For that reason, we have to estimate the parameters of this distribution by using the real data of Brazilian Market to check whether the candidate is a good substitute for the geometric Brownian motion which, as already mentioned, has several flaws. Next, we will present this estimation.

\subsection{Parameter estimates.}

We estimate the hyperbolic distribution parameters using the Hyp program developed by Blæsid and Sørensen. The estimation is made by maximum likelihood with the hypothesis of the independent and identically distributed random variables. See Blæsid and Sørensen (1992) for the description of the algorithm. The results are shown in Table 1.

Based on the construction of the hyperbolic distribution, it is necessary to have a great number of data in the sample so that we can obtain a reasonable estimate of tail behavior. We should expect this restriction not to influence the use of the hyperbolic model in finance due to samples with a large number of data. 
Lévy processes and the Brazilian market

Table 1: Estimation of hyperbolic distribution parameters.

\begin{tabular}{lcccc}
\hline & $\widehat{\alpha}$ & $\widehat{\beta}$ & $\widehat{\delta}$ & $\widehat{\mu}$ \\
\hline Ibovespa (G1) & 49.53 & -3.939 & $1.638 \mathrm{E}-09$ & $3.689 \mathrm{E}-03$ \\
Ibovespa (G2) & 66.59 & -7.430 & $5.937 \mathrm{E}-09$ & $5.092 \mathrm{E}-03$ \\
Telebrás (G1) & 43.67 & -2.704 & $8.488 \mathrm{E}-03$ & $4.012 \mathrm{E}-03$ \\
Telebrás (G2) & 56.37 & -4.136 & $7.291 \mathrm{E}-03$ & $4.920 \mathrm{E}-03$ \\
Petrobrás (G1) 42.58 & -1.804 & $9.359 \mathrm{E}-03$ & $2.578 \mathrm{E}-03$ \\
Petrobrás (G2) 54.95 & -3.437 & $7.488 \mathrm{E}-03$ & $4.952 \mathrm{E}-03$ \\
\hline
\end{tabular}

Like Eberlein and Keller (1995), we used the $\chi^{2}$ test to analyze the adherence of data to the hyperbolic distribution. The estimated $\hat{\chi}^{2}$ values and the critical value of variable $\chi_{\nu}^{2}$ with a significance of $1 \%$ are shown in Table 2. The degrees of freedom are given by $\nu=k-1-m$, where $k$ is the number of classes and $m=4$ is the number of estimated parameters. In all cases, we have $\hat{\chi}^{2}<\chi_{\alpha=1 \%}^{2}$, which does not let us reject the null hypothesis of the hyperbolic behavior.

Table 2: $\chi^{2}$ with hyperbolic distribution for $H_{0}$

\begin{tabular}{lrl}
\hline & $\hat{\chi}^{2}$ & $\chi_{\alpha=1 \%}^{2}$ \\
\hline Ibovespa (G1) & 17.250 & 21.666 \\
Ibovespa (G2) & 9.283 & 18.475 \\
Telebrás (G1) & 12.070 & 20.090 \\
Telebrás (G2) & 9.687 & 20.090 \\
Petrobrás (G1) & 13.601 & 18.475 \\
Petrobrás (G2) & 16.937 & 18.475 \\
\hline
\end{tabular}

It is important to emphasize that if we change the significance level to $5 \%$, we will reject the null hypothesis in 2 cases, which would not let us obtain more robust results. 
José Fajardo Barbachan, Andrés Ricardo Schuschny and André de Castro Silva

Figure 5 shows the histograms of data and their estimated density. The graphs are represented on a logarithmic scale so that the adjustment can be viewed more easily. If the returns follow the hyperbolic distribution, their log histogram should present an increasing and then a decreasing line. The simplest curve with such behavior is the hyperbola. We note that the theoretical curves are in agreement with the observations, especially regarding the data that are closer to the mean. However, for tail data, there are off-curve points. This could make us think that the use of these distributions would be inappropriate. If we find the Kolmogorov distance (KD) and Anderson-Darling statistic $(\mathrm{AD})^{5}$ we can observe that the adjustment obtained through the hyperbolic distribution is far better than that made via normal distribution, as shown in the following table.

\begin{tabular}{lllll} 
& $D K($ hyp $)$ & $D K($ normal $)$ & $A D($ hyp $)$ & $A D($ normal $)$ \\
\hline Ibovespa (G1) & 0.0243382 & 0.0892196 & 0.649622 & 4518.23 \\
Ibovespa (G2) & 0.032024 & 0.10132 & 0.69617 & 2043.33 \\
Telebrás (G1) & 0.020799 & 0.075921 & 3.3639 & 96156.3 \\
Telebrás (G2) & 0.015393 & 0.076458 & 0.355854 & 782.979 \\
Petrobrás (G1) & 0.015592 & 0.071611 & 3.3639 & 96156.3 \\
Petrobrás (G2) & 0.033111 & 0.067329 & 0.355854 & 4550.97
\end{tabular}

This can also be seen in Figure 5, since the graph of lognormal density would be a parabola, which would leave more points off the curve. In case of the stable Paretian distribution, we would have a straight line, which would also be an extreme case.

${ }^{5}$ See appendix.

Brazilian Review of Econometrics

21 (2) November 2001 
Lévy processes and the Brazilian market
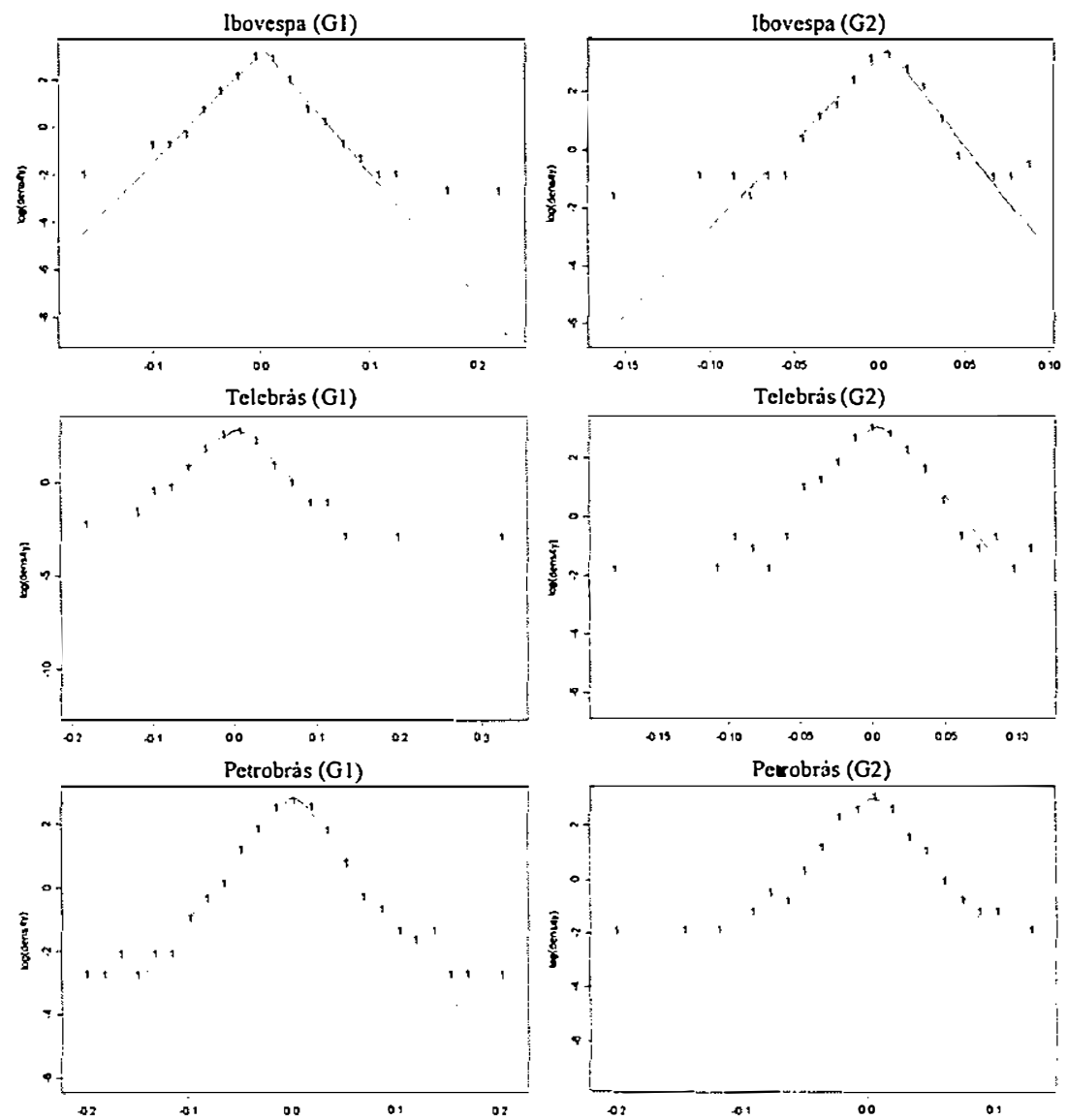

Figure 5: Log-log scale histograms for returns

The coefficients estimated according to parametrization (22) are shown in Table 3 . We observe that the coefficients take the distribution to a region that is far from the boundary with normal distribution, as shown in Figure 4. 
José Fajardo Barbachan, Andrés Ricardo Schuschny and André de Castro Silva

Table 3: Shape triangle parameters.

\begin{tabular}{lrc}
\hline & $\widehat{\xi}$ & $\widehat{\chi}$ \\
\hline Ibovespa (G1) & 1.0000 & -0.0795 \\
Ibovespa (G2) & 1.0000 & -0.1116 \\
Telebrás (G1) & 0.8544 & -0.0529 \\
Telebrás (G2) & 0.8422 & -0.0618 \\
Petrobrás (G1) & 0.8457 & -0.0358 \\
Petrobrás (G2) & 0.8420 & -0.0527 \\
\hline
\end{tabular}

\subsection{Scale Invariance.}

To analyze the scale invariance, we have to use a result obtained by Barndorff-Nielsen and Prause (2001), which shows that distributions with semi-heavy tails have an apparent invariance; therefore, as previously observed, hyperbolic distributions have semi-heavy tails and, consequently, do not have scale invariance.

\section{Conclusions.}

Based on the previous analysis, we may conclude that the adjustment obtained through the hyperbolic distribution is good and as a consequence better than the adjustment made via normal distribution and via the stable Paretian distribution, as the former has a very light tail and the latter, a very 'fat' tail. Hyperbolic distributions have semi-heavy tails, which allow better adjustment for some series at a given significance level. In addition, we noted that the parameters do not have a clear meaning; however, by changing parameters, it is possible to understand $\xi$ and $\chi$ asymptotically as kurtosis and asymmetry, respectively.

Hopefully, we expect to have achieved our goal by showing some empirical evidence and discussing the use of continuous time distributions for the analysis of the Brazilian market. 
6. Appendix.

\subsection{Power Law.}

We say that $N$ has a potential behavior and follows a power law when

$$
N(s) \propto s^{\alpha}
$$

\subsection{Bessel Functions.}

The modified Bessel function $\left(K_{\lambda}\right)$ has the following integral representation

$$
K_{\lambda}(x)=\frac{1}{2} \int_{0}^{\infty} y^{\lambda-1} \exp \left(-\frac{x}{2}\left(y+y^{-1}\right)\right) d y, \quad x>0,
$$

Some basic properties:

1.

$$
K_{\lambda}(x)=K_{-\lambda}(x)
$$

2.

$$
K_{\lambda+1}(x)=\frac{2 \lambda}{x} K_{\lambda}(x)+K_{\lambda-1}(x)
$$

\subsection{Generation of Lévy Processes.}

Take $\left\{X_{t}\right\}_{t \geq 0}$ as the Lévy process generated by the hyperbolic distribution with hyp density, i.e., the process with independent and stationary increments such that $X_{0}=0$ and the distribution of $X_{1}$ 
José Fajardo Barbachan, Andrés Ricardo Schuschny and André de Castro Silva

has hyp density. When $\mu=0$, Eberlein and Keller (1995) called $\left\{X_{t}\right\}_{t \geq 0}$ Hyperbolic Lévy motion.

The mean and variance are finite, and this was previously calculated; however, we can observe that $E\left|X_{t}\right|^{p}<\infty, \forall t \geq 0,(p \geq 1)$.

\section{$6.4 \mathrm{KD}$ and $\mathrm{AD}$.}

Take $\left\{X_{t}\right\}_{t \geq 0}$ as the Lévy process generated by

Let us suppose that $F(x)$ is an accumulated distribution function, therefore, Kolmogorov distance is given by

$$
D K=\max _{x \in \mathbb{R}}\left|F_{\text {empirical }}(x)-F_{\text {estimated }}(x)\right|,
$$

and Anderson-Darling statistic is given by:

$$
A D=\max _{x \in \mathbb{R}} \frac{\left|F_{\text {empirical }}(x)-F_{\text {estimated }}(x)\right|}{\sqrt{F_{\text {estimated }}(x)\left(1-F_{\text {estimated }}(x)\right)}}
$$

Submitted in December 1999. Revised in March 2002.

\section{References}

Abramowitz, M. \& I.A. Stegun 1968. Handbook of Mathematical Functions. Dover, New York.

Barndorff-Nielsen, O.E. 1977. "Exponentially decreasing distributions for the logarithm of particle size". Proceedings of the Royal Society London, A 353:401-419.

Barndorff-Nielsen, O.E. 1978. "Hyperbolic distributions and distributions on hyperbolae". Scandinavian Journal of Statistics, 5:151-157. 
Barndorff-Nielsen, O.E. 1997. "Processes of Normal Inverse Gaussian Type". Working Paper Series no. 1, Center for Analytical Science, Aarhus School of Business.

Barndorff-Nielsen, O.E., P. Blæsid, J.L. Jensen, \& M. K. Sørensen 1985. "The fascination of sand", in Atkinson, A.C. Fienberg, S.E. (eds.), A celebration of statistics, 57-87, Springer: New York.

Barndorff-Nielsen, O.E. \& K. Prause 2001. "Apparent Scaling". Finance and Stochastics, 5:103-113.

Blæsid, P. \& M. K. Sørensen 1992. "Hyp- a computer program for analyzing data by means of the hyperbolic distribution", Research Report no. 248, Department of Theoretical Statistics, University of Aarhus.

Duarte J., A.M. \& B.V.M. Mendez 1999. "Robust Estimation for ARCH Models". Brazilian Review of Econometrics, 19:139-180.

Eberlein, E. \& U. Keller 1995. "Hyperbolic distributions in finance", Bernoulli , 1:281-299.

Eberlein, E., U. Keller \& K. Prause 1998. "New insights into smile, mispricing and value at risk: the hyperbolic model", Working Paper n. 39 (revised version), Center for Data Analysis and Model Building, University of Freiburg.

Fama, E. 1965. "The behaviour of stock market prices", The Journal of Business, 38:134-105.

Issler, J.V. 1999. "Estimating and Forecasting the Volatility of Brazilian Finance Series Using ARCH Models". Brazilian Review of Econometrics, 19:15-56.

Kuchler, U., K. Neumann, M. Sørensen \& A. Streller 1994. "Stock returns and Hyperbolic distributions", Discussion paper 23, Sonderforschungsbereich 373, Humboldt-Universität zu Berlin. 
José Fajardo Barbachan, Andrés Ricardo Schuschny and André de Castro Silva

Lévy, P. 1937. "Théorie de l'Addition des Variables Aléatories, Gauthier-Villars, Paris.

Mandelbrot, B. 1963. "The variation of certain speculative price", The Journal of Business, 36:4394-419.

Mandelbrot, B. 1997. "Fractals and Scaling in Finance". SpringerVerlag.

Mandelbrot, B. \& H. Taylor 1967. " On the distributions of stock price differences". Operations Research 15:1057-1062.

Mantegna, R. 1995. "Scaling Behaviour in the Dynamics of an Economic Index". Nature, 376:6535,46-49

Mazuchelli, J. \& H. S. Migon 1999. "Modelos GARCH Bayesianos:

Métodos Aproximados e Aplicaçōes". Brazilian Review of Econometrics, 19:1, 111-138.

Pereira, P.L.V., L.K. Hotta, L.A.R. Souza \& N.M.C.G. Almeida, 1999. "Alternative Models to Extract Asset Volatility: A Comparative Study". Brazilian Review of Econometrics, 19:1, 57109. 
